\title{
Hamartoma mesenquimatoso de la pared torácica: presentación de un caso
}

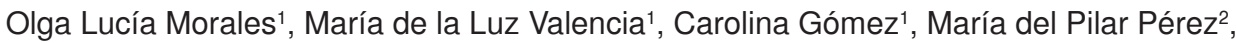 \\ Emilio Sanín ${ }^{3}$, Luz Marina Vásquez ${ }^{4}$ \\ Sección de Neumología Pediátrica, Grupo Pediaciencias, Departamento de Pediatría, Facultad de Medicina, \\ Universidad de Antioquia, Medellín, Colombia \\ 2 Departamento de Patología, Facultad de Medicina, Universidad de Antioquia, Medellín, Colombia \\ 3 Departamento de Radiología, Facultad de Medicina, Universidad de Antioquia, Medellín, Colombia \\ ${ }^{4}$ Servicio de Neonatología, Hospital Universitario San Vicente de Paúl, Medellín, Colombia \\ Institución donde se realizó el trabajo: \\ Hospital Universitario San Vicente de Paúl, Medellín, Colombia
}

El hamartoma mesenquimatoso de la pared torácica es un tumor benigno extremadamente raro. Hasta el momento hay, aproximadamente, 80 casos reportados en la literatura mundial; la mayoría se manifiesta desde el nacimiento por la presencia de una masa palpable, no dolorosa, usualmente unilateral, en la pared torácica.

Los síntomas respiratorios son secundarios a la compresión extrínseca sobre el parénquima pulmonar y su gravedad depende del tamaño y la localización de la lesión. Los hallazgos radiológicos son característicos pero el diagnóstico definitivo es histológico.

Describimos el caso de un paciente de cuatro meses de edad, en quien se realizó diagnóstico de hamartoma mesenquimatoso de la pared torácica; el paciente presentaba las manifestaciones desde el nacimiento.

Se hace énfasis en las opciones de tratamiento, considerando el manejo expectante como una alternativa válida por las características benignas de este tumor, por la considerable morbilidad posterior a grandes tratamientos quirúrgicos y por la alta probabilidad de presentar regresión espontánea.

Palabras clave: hamartoma, pared torácica, costillas, tórax, lactante.

\section{Chest wall mesenchymal hamartoma: a case report}

Chest wall mesenchymal hamartoma is an extremely rare benign tumor. Approximately 80 cases have been reported in the literature. Most tumors are manifested at birth with a painless palpable mass of the chest wall, usually unilateral. Respiratory symptoms result from extrinsic compression of the pulmonary parenchyma, and the severity of the symptoms will depend on the size and location of the lesion. Imaging features are characteristic, but definitive diagnosis is histological. Herein, a case is described of a four month old infant with diagnosis of chest wall mesenchymal hamartoma, manifested at birth. Different treatment options are described, including expectations from tumor management, the possibility of spontaneous regression, and the morbidity associated with the surgical option.

Key words: hamartoma, thoracic wall, ribs, torax, infant.

El hamartoma mesenquimatoso de la pared torácica constituye una lesión tumoral benigna extremadamente rara. Corresponde a $0,03 \%$ de los tumores óseos primarios y, hasta la fecha, hay, aproximadamente, 80 casos reportados en la literatura médica (1).

Fue descrito por primera vez en 1962, como un tumor mesodérmico benigno que se origina en las

\section{Correspondencia:}

Carolina Gómez, Calle 80 №34-36, apartamento 506, Medellín, Colombia.

Teléfono: (574) 250 4742; fax: (574) 4166232

carogldm@hotmail.com

Recibido: 10/03/09; aceptado:15/10/09 costillas (2). Se han usado varios términos para denominarlo: hamartoma de la pared torácica, hamartoma condromatoso gigante, hamartoma cartilaginoso y hamartoma cartilaginoso vascular. En la década de los 80 se usaba preferentemente el término mesenquimoma, hasta 1986, cuando Odell y Benjamin empezaron a llamarlo hamartoma mesenquimatoso de la pared torácica, término actualmente más aceptado (2).

Por lo general, es evidente desde el nacimiento o en los primeros meses de vida (3), aunque puede identificarse incidentalmente en radiografías torácicas en personas mayores. En una serie de casos, se encontró que $75 \%$ de los casos se 
manifiestan antes del primer año de vida por la presencia de masa visible sobre la pared torácica; sin embargo, hasta $60 \%$ pueden ser asintomáticos (4). Los síntomas respiratorios son secundarios a la compresión extrínseca sobre el parénquima pulmonar y su gravedad depende del tamaño y la localización de la lesión (5). La mayoría de los casos son unilaterales, principalmente en el hemitórax derecho, pero hay descritos casos bilaterales (6). Tiene una relación de hombre a mujer de 2 a 1 (7).

Esta masa tumoral emerge de las costillas y está constituida por una proliferación benigna del tejido esquelético, con gran componente cartilaginoso y quistes óseos aneurismáticos secundarios, sin invasión ni metástasis. En la histología se observa predominantemente tejido cartilaginoso con células gigantes osteoclásticas, osificación encondral y maduración trabecular ósea (8).

El hamartoma mesenquimatoso de la pared torácica con frecuencia es erróneamente interpretado como condrosarcoma, osteosarcoma o mesenquimoma maligno, por su tendencia al crecimiento progresivo, especialmente en el primer año de vida, a tal punto que, incluso, se han tratado en algunas ocasiones con radioterapia y quimioterapia, terapias que no han demostrado ventajas adicionales (2).

El hamartoma mesenquimatoso de la pared torácica se caracteriza por tener un crecimiento progresivo; sinembargo, puede presentar regresión espontánea o, menos frecuentemente, sufrir una trasformación maligna (2). Habitualmente, el crecimiento se detiene cerca del primer año de vida, lo que hace innecesario el tratamiento quirúrgico en la mayoría de los casos (9).

El diagnóstico definitivo es histológico; sin embargo, el hallazgo radiológico es característico: lesión expansiva con origen en una o más costillas, con erosión y destrucción de las mismas y una masa de tejidos blandos con calcificaciones burdas de localización extrapleural. Con los métodos de imaginología por cortes se observa una masa de origen costal, expansión ósea, destrucción de las costillas adyacentes y masa de tejidos blandos que compromete desde el tejido celular subcutáneo hasta los planos musculares más profundos. Se pueden observar imágenes quísticas, con nivel líquido-líquido, por sangrado dentro de los quistes óseos aneurismáticos secundarios. Este hallazgo en un neonato es patognomónico de hamartoma mesenquimatoso de la pared torácica (4).

\section{Presentación del caso clínico}

Se trata de un paciente de sexo masculino que nació por parto vaginal sin complicaciones a las 38 semanas de gestación, con buena adaptación neonatal y peso adecuado para la edad. En el examen físico se encontró una masa torácica latero-posterior derecha de $5,0 \mathrm{~cm} \times 7,0 \mathrm{~cm}$, adherida a los planos profundos, dura, sin cambios en la coloración de la piel, y sin otros hallazgos anormales.

Se obtuvieron radiografía de tórax (figura 1), tomografía contrastada de tórax (figuras 2 y 3 ) y biopsia de la lesión (figura 4).

La histología describió una tumoración benigna conformada por proliferación de componentes heterólogos dispuestos en forma desorganizada, constituidos por músculo estriado, tejido fibroconjuntivo, linfocitos, hueso maduro, cartílago y células gigantes, hallazgos correspondientes al hamartoma mesenquimatoso de la pared torácica (figura 4).

En su evolución, el paciente presentó neumonía hospitalaria en la primera semana de vida, la cual respondió adecuadamente al tratamiento antibiótico sin requerir asistencia respiratoria ni medicamentos inotrópicos. Fue dado de alta a los 20 días de vida en muy buenas condiciones, sin necesidad de oxígeno domiciliario y con un plan de seguimiento ambulatorio.

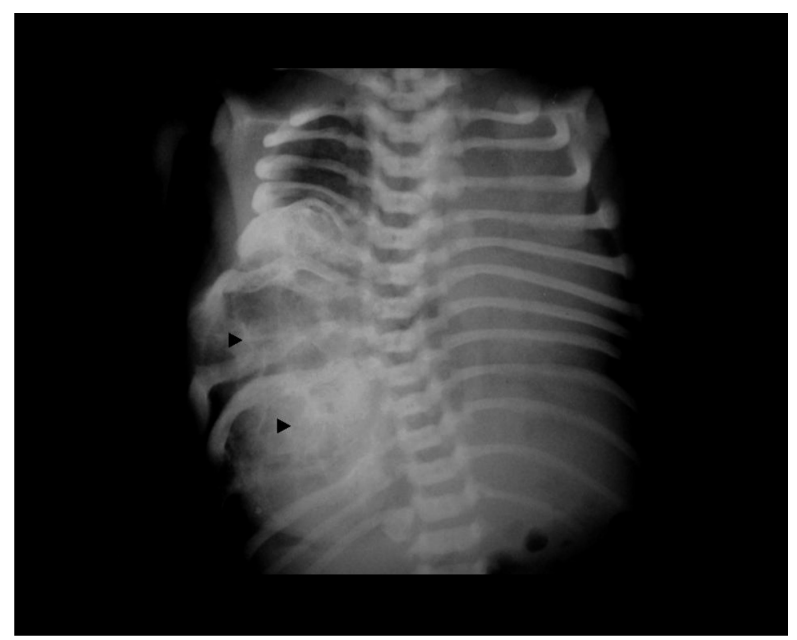

Figura 1. Radiografía de tórax en la que se evidencia deformidad de la reja costal derecha por la presencia de una gran masa que tiene calcificación con patrón de mineralización osteoide y condroide (puntas de flecha) que, a la vez, erosiona y distorsiona los arcos costales, con disminución del volumen pulmonar del mismo lado y desviación del mediastino hacia el lado izquierdo. 


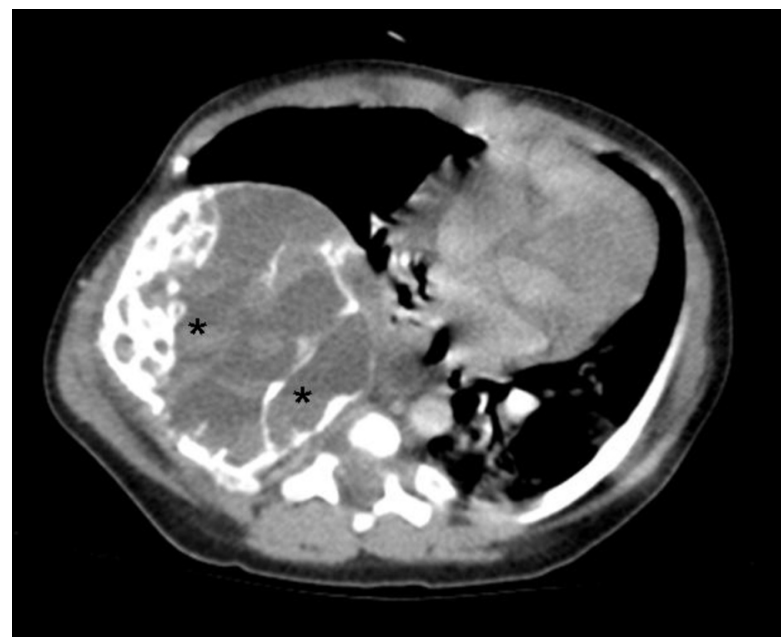

Figura 2. Tomografía contrastada de tórax. Corte axial en el que se observa gran lesión extrapleural que destruye los arcos costales posteriores derechos, la cual tiene componente de tejidos blandos y calcificaciones con patrón de mineralización condroide y osteoide e imágenes quísticas con niveles líquidolíquido (asterisco), por sangrado dentro de los quistes óseos aneurismáticos secundarios. Hay compresión del parénquima pulmonar adyacente y desplazamiento del mediastino hacia el lado contrario.

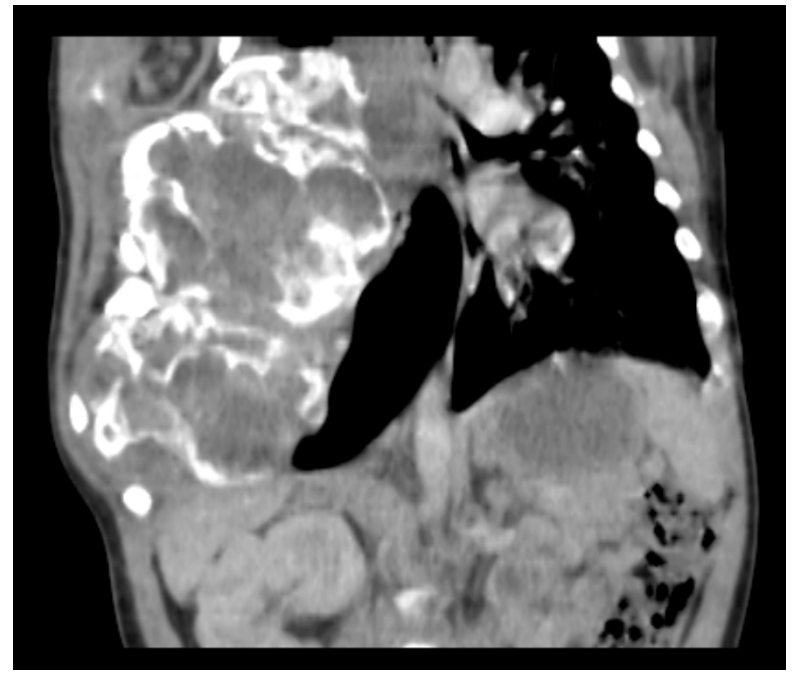

Figura 3. Tomografía contrastada de tórax. Reconstrucción coronal, en la que se observa la extensión en sentido longitudinal, con afectación de casi la totalidad de la pared torácica derecha.

Al mes de vida se hospitalizó por presentar episodio de bronquiolitis moderada, con resultados negativos para virus sincitial respiratorio. La masa torácica anteriormente descrita no había presentado cambios con respecto al nacimiento (figura 5a).

El manejo para este lactante seguirá siendo conservador hasta que su condición clínica amerite un tratamiento definitivo o su peso permita

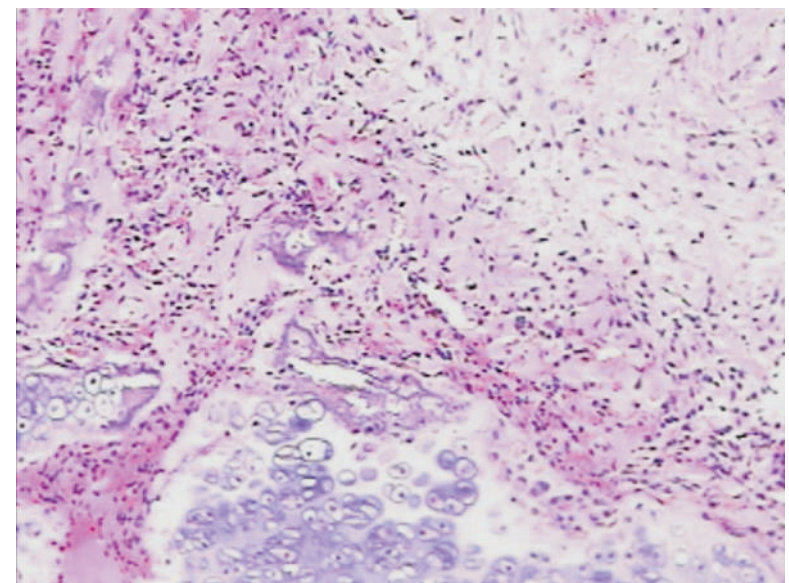

Figura 4. Biopsia de masa torácica que muestra la proliferación de componentes heterólogos constituidos por músculo estriado, tejido fibroconjuntivo, linfocitos, hueso maduro y cartílago. Este material está dispuesto en forma desorganizada y es indicativo de hamartoma mesenquimatoso de la pared torácica. Hematoxilina eosina, 10X.

técnicas menos invasivas, como la termoablación con radiofrecuencia. Dadas las característica benignas de la lesión, no se realizó radioterapia ni quimioterapia.

Al momento de terminar este manuscrito, el paciente cumple ocho meses de vida, tiene adecuado crecimiento y desarrollo psicomotor, no ha vuelto a presentar síntomas respiratorios, no ha requerido oxígeno domiciliario, la masa torácica no ha presentado crecimiento adicional (figura $5 b$ ) y está en seguimiento ambulatorio por el Grupo de Neumología Pediátrica.

\section{Discusión}

El hamartoma mesenquimatoso de la pared torácica es una lesión tumoral muy rara, con pocos casos reportados en la literatura médica (10). Se manifiesta principalmente por una masa en la pared torácica, con diferentes grados de compromiso sobre la función respiratoria, desde el estado asintomático, hasta la falla respiratoria (10).

En el estudio de un niño con masa en la pared torácica deben considerarse, entre las opciones etiológicas, las fracturas claviculares, las lesiones óseas primarias benignas (displasia fibrosa, quiste óseo aneurismático, osteocondroma y hamartoma mesenquimatoso de la pared torácica), las lesiones óseas primarias malignas (osteoblastoma, osteosarcoma y sarcoma de Ewing), el teratoma maligno, el neuroblastoma congénito y las malformaciones vasculares. Las causas secundarias de deformidad de la pared 

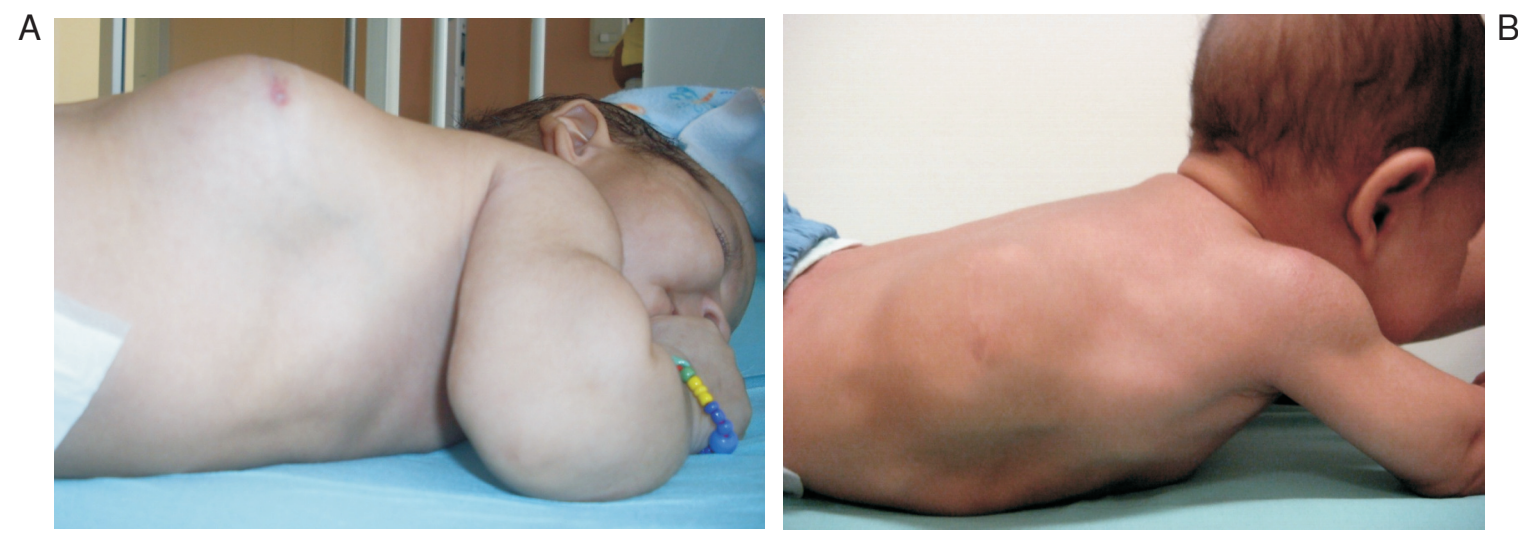

Figura 5. A. Paciente al mes de vida, que presenta una masa torácica posterior y lateral que causa deformidad de la caja torácica. B. Paciente a los ocho meses, sin progresión del tamaño de la masa.

torácica incluyen enfermedades sistémicas como el síndrome de Marfan, la osteopetrosis y las displasias óseas (11).

En años recientes, el uso rutinario de la ecografía obstétrica ha incrementado la identificación intrauterina del hamartoma mesenquimatoso de la pared torácica. Inicialmente en estas lesiones se describe un periodo de rápido crecimiento que cede posteriormente $y$, en ocasiones, tiene regresión espontánea (12). La importancia del diagnóstico prenatal radica en la preparación que el equipo médico debe tener para atender estos pacientes, anticipando la posible presencia de hipoplasia pulmonar con sus consecuencias y, además, poder ofrecerles un manejo conservador hasta obtener el diagnóstico histológico definitivo y manejo según el caso.

Describimos el caso de un paciente con diagnóstico clínico, radiológico e histológico de hamartoma mesenquimatoso de la pared torácica, con gran extensión de la lesión tumoral, tanto intratorácica como extratorácica, y una afectación significativa de los arcos costales, en quien, dada la significativa morbilidad que podría ocasionar el tratamiento quirúrgico, se decidió un manejo conservador de acuerdo con su evolución en el tiempo.

Las recomendaciones de tratamiento son variables. Hay poca controversia respecto al tratamiento de pacientes con hamartoma mesenquimatoso de la pared torácica que cursa con alteración respiratoria, ya que ellos deben recibir manejo quirúrgico (13) con escisión completa en bloque.

La discusión se centra en el tratamiento de los pacientes asintomáticos, ya que existe la opción de un manejo conservador, especialmente, en los casos que impliquen grandes mutilaciones quirúrgicas (8). Sin embargo, en la mayoría de los reportes de casos publicados, se observa una tendencia a preferir el manejo quirúrgico, lo que se explica por la dificultad existente para distinguir esta lesión de una maligna, por la posibilidad, aunque mínima, de trasformación maligna, por su usual crecimiento progresivo y por la corrección de la deformidad ósea (14).

Por otro lado, algunas publicaciones cuestionan la necesidad de someter a un paciente asintomático a grandes cirugías que causan morbilidad importante, producida por la resección radical de la pared torácica, con deformidad progresiva de la misma y escoliosis secundaria a la resección de un número significativo de costillas, por el riesgo de sangrado debido al significativo componente vascular del tumor (15), además de la inestabilidad de la pared torácica, que aumenta el riesgo de muerte y la posibilidad de nuevo crecimiento de la masa cuando su resección no es total.

La tercera opción de tratamiento, tal vez un poco más prudente, sugiere que, debido a las características benignas de este tumor y dadas las posibilidades de que se presente una reducción en su tamaño con regresión espontánea en el tiempo, como se ha documentado en algunos casos (16), se ha sugerido posponer el tratamiento quirúrgico hasta cuando esté claramente indicado (17).

Otra opción de tratamiento es la ablación tumoral con radiofrecuencia, una técnica poco invasiva, en la cual se realiza ablación localizada del tejido patológico, por medio de electrodos percutáneos y con la guía de la tomografía computadorizada. Esta técnica ha sido usada, particularmente, en el tratamiento del osteoma osteoide y 
del condroblastoma epifisiario y, por la similitud histológica, se ha extendido al tratamiento del hamartoma mesenquimatoso de la pared torácica (1).

A pesar de su alarmante apariencia, el hamartoma mesenquimatoso de la pared torácica es una lesión benigna que puede manejarse conservadoramente si no hay compromiso respiratorio o acentuada deformidad de la pared torácica. Para el diagnóstico definitivo, se debe hacer una buena correlación entre los estudios radiológicos y los histológicos.

\section{Agradecimientos}

A los departamentos de Pediatría y Puericultura, Patología y Radiología de la Universidad de Antioquia; al Área de Neumología Infantil de la Universidad de Antioquia; al paciente y a su familia.

\section{Conflicto de intereses}

Durante la realización de este artículo, ninguno de los autores tuvo vinculación con alguna actividad que pudiera implicar conflictos de interés.

\section{Financiación}

No se recibieron recursos de ninguna entidad nacional o internacional.

\section{Referencias}

1. Bertocchini A, Falappa P, Accinni A. Radiofrequency thermoablation in chest wall mesenchymal hamartoma of an infant. Ann Thorac Surg. 2007;84:2091-3.

2. Odell JM, Benjamin DR. Mesenchymal hamartoma of chest wall in infancy: natural history of two cases. Pediatr Pathol.1986;5:135-46.

3. Shamberger RC, Grier HE, Weinstein HJ, Atayde AR, Tarbell NJ. Chest wall tumors in infancy and childhood. Cancer. 1989;63:774-85.

4. Groom KR, Murphey MD, Howard LM, Lonergan GJ, Rosado-de- Christenson ML, Torop AH. Mesenchymal hamartoma of the chest wall: Radiologic manifestations with emphasis on cross-sectional imaging and histopathologic comparison. Radiology. 2002;222:205-11.

5. Nogues A, Gervas C, Oñativia A, Collado V. Benign costal mesenchymal hamartoma in a neonate. Pediatr Radiol. 2003;33:221-2.

6. Lisle DA, Ault DJ, Earwaker JW. Mesenchymal hamartoma of the chest wall in infants: Report of three cases and literature review. Australas Radiol. 2003;47:78-82.

7. Andiran F, Ciftci AO, Senocak ME, Akcören Z, Gögüs S. Chest wall hamartoma: An alarming chest lesion with a benign course. J Pediatr Surg. 1998;33:727-9.

8. Cameron D, Ong TH, Borzi P. Conservative management of mesenchymal hamartomas of the chest wall. J Pediatr Surg. 2001;36:1346-9.

9. Altaner S, Yoruk Y, Bilgi S, Puyan FO, Doganay L, Kutlu $\mathrm{K}$. Multifocal mesenchymal hamartoma of the chest wall. Respirology. 2006;11:334-8.

10. Shimotake T, Fumino S, Aoi S, Tsuda T, Iwai N. Respiratory insufficiency in a newborn with mesenchymal hamartoma of the chest wall occupying the thoracic cavity. J Pediatr Surg. 2005;40:E13-6.

11. Donnelly LF, Frush DP. Abnormalities of the chest wall in pediatric patients. AJR Am J Roentgenol. 1999;173:1595601.

12. Freeburn AM, McAloon J. Infantile chest hamartoma-case outcome aged 11. Arch Dis Child. 2001;85:244-5.

13. Brand T, Hatch El, Schaller RT, Stevenson JK, Arensman RM, Schwartz MZ. Surgical management of the infant with mesenchymal hamartoma of the chest wall. J Pediatr Surg. 1986;21:556-8.

14. Kerrey B, Reed J. A neonate with respiratory distress and a chest wall deformity. Pediatr Emerg Care. 2007;23:565-9.

15. Ayala AG, Ro JY, Bolio-Solis A, Hernandez-Batres F, Eftekhari F, Edeiken J. Mesenchymal hamartoma of the chest wall in infants and children: a clinicopathologic study of five patients. Skeletal Radiol. 1993;22:569-76

16. Campbell AN, Wagget J, Mott MG. Benign mesenchymoma of the chest wall in infancy. J Surg Oncol. 1982;21:267-70.

17. Kadell BM, Coulson WF, Desilets DT, Fonkalsrud EW. Congenital atypical benign chondroblastoma of a rib. $\mathrm{J}$ Pediatr Surg. 1970;5:46-52. 